$\underline{\text { Review Article }}$

\title{
AN OVERVIEW OF VARIOUS SCALES USED IN CAUSALITY ASSESSMENT OF ADVERSE DRUG REACTIONS
}

\author{
ADUSUMILLI PRAMOD KUMAR ${ }^{*}$, DHARINI BHOOPATHI ${ }^{2}$, HARIPRIYA SUNKARA¹, SRI HARSHA CHALASANI ${ }^{3}$ \\ 1Department of Pharmacy Practice, Chebrolu Hanumaiah Institute of Pharmaceutical Sciences, Guntur, Andhrapradesh, ${ }^{2}$ Adverse Drug \\ Reaction Monitoring Center, SDS Tuburculosis Research Centre and Rajiv Gandhi Institute of Chest Diseases, Bengaluru, Karnataka, \\ ${ }^{3}$ Faculty of Pharmacy, JSS Academy of Higher Education and Research, Mysuru, Karnataka.
}

Email: pramodkumar.adusumilli@gmail.com

Received: 20 Feb 2020, Revised and Accepted: 30 Mar 2020

\section{ABSTRACT}

Establishing a relationship of causality between the medications received and the events occurred utilizing causality assessment scale is much needed to reduce the occurrence of Adverse Drug Reactions (ADRs) and to prevent exposure of patients towards additional drug hazards. Causality assessment can be defined as the determination of chance, whether a selected intervention is the root cause of the adverse event observed. The causality assessment is the responsibility of either a single expert or an established committee. As it is a common phenomenon of variable perception of knowledge and experience by each expert, there is a high possibility of disagreement and inter-individual variability on assessment. Many of the causality assessment methods have their advantages and disadvantages. However, no single scale has been adopted as standardized and considered for uniform acceptance.

Keywords: Pharmacovigilance, Causality assessment scales, Adverse drug reactions

(C) 2020 The Authors. Published by Innovare Academic Sciences Pvt Ltd. This is an open access article under the CC BY license (http://creativecommons.org/licenses/by/4.0/) DOI: http://dx.doi.org/10.22159/ijpps.2020v12i5.37209. Journal homepage: https://innovareacademics.in/journals/index.php/ijpps

\section{INTRODUCTION}

Pharmacovigilance is an integral branch of the science which is related to collection, detection, assessment, understanding, and prevention of adverse effects or any other problems related to drug use [1]. World Health Organization (WHO) defined the ADR as "response to a drug, which is noxious and unintended, and which occurs at doses normally used in man for prophylaxis, diagnosis or therapy of disease, or for the modification of physiological function [2]. This definition excludes overdose, abuse of the drug, treatment failure and drug administration errors. The female gender, age (very young and very old), multiple medications and the physiological state of renal and liver function, breastfeeding, pregnancy, and alcohol intake are considered as the important risk factors for ADRs [3].

Adverse drug effects not only entirely intrude with the patient's compliance towards the treatment, but they also additionally increase morbidity and mortality, which also reflects on the financial burden of the society. Of the total ADRs estimated 6.7\% (range 1.2$24.1 \%$ ) was identified in hospitalized patients and of that $0.32 \%$ $(0.1-0.85 \%)$ being fatal [4]. In a South Indian study, about $3.7 \%$ of the total hospitalized patients were observed to be suffering from ADRs, and among them, $1.3 \%$ were fatal. About $0.7 \%$ of the hospitalizations were because of ADRs [5]. A study conducted by Arulmani et al. reported that among the collected ADR reports in the hospital, 3.4\% of ADR related cases were approved to require hospitalization and 3.7\% ADRs developed in the patients during the period of hospitalization [6]. In recent times, the meta-analysis by Alhawassi et al. [7]. Which included 14 studies found $10.0 \%$ (95\%CI 7.2-12.8\%) hospitalizations because of ADRs, amidst inpatients over $65 \mathrm{y}$ of age; this study determined that hospitalization of one in ten is because of ADRs. Data indicates that $19.18 \%$ higher death rate and $8.25 \%$ increased length of hospital stay among inpatients that experience ADRs. It has been estimated that patients with ADRs suffer an average increase of $19.86 \%$ in their total medical costs [8].

In a systematic review conducted on 25 observational studies, the percentage of hospitalizations due to ADRs was $4 \%$ among children, $6 \%$ among adults, and $11 \%$ among older adults [9-10]. The potential to suspect or identify adverse events with plausible explanations and standard measures to avoid inappropriate management of ADRs is expected from clinicians and clinical pharmacists. Establishing or evaluating likelihood between the adverse event and the drug through causality assessment is much needed to reduce the occurrence of ADRs and to prevent exposure of patients towards additional drug hazards [11]. It is a key element of Pharmacovigilance and contributes to evaluate risk-benefit profiles and identify signals, thereby assisting in regulatory decision making [13].

Informal causality assessment of ADRs is in general practice by healthcare professionals to conclude decisions regarding therapy management [12]. Algorithms should provide more objective decision on causality rather than theoretical explanation in identifying adverse events during therapy. The four basic principles underlying the objective causal assessment include-1) temporal eligibility, 2) dechallenge and outcome, 3) rechallenge and outcome, and 4) confounding factors [14]. The current review article focuses on the merits and demerits of different assessment scales and briefs the use of a specific scale for a given clinical practice setup.

\section{Methods of causality assessment}

To characterize ADRs, research workers identified several causality assessment methods based on different criteria such as analogous scales, theorems, probability scales, algorithms, etc. however, interrater and intra-rater variability are wide as there is no predefined diagnostic criteria or classifications $[15,16]$.

Until now, not a single causality assessment scale has been accepted and adopted universally due to variability and inconsistency in reproducibility and validity [17]

A High incidence of ADRs arises during the treatment at tertiary care hospitals [22]. Hence causality assessment at such instances contributes to i) early identification of ADRs and minimization of further complications ii) Optimized therapy [23]; iii) new strategical treatment to avoid recurrence iv) Cost minimization by reducing prolonged hospitalization [24].

Source of information and search strategy for identifying relevant studies

To ensure a comprehensive research review of the subject, we performed searches using terms such as medical subject headings (MeSH) and key text words, such as "causality assessment scales," "causality evaluation", "methods for causality assessment", "tools for causality assessment", "algorithm for causality" published till 2018. 
Thus, the abstracts of published studies with relevant information on the drug information centers were identified. These terms were used individually and in combination to ensure an extensive literature search. Relevant articles were selected and collated based on the broader objective of the review. This was achieved by searching databases, including SCOPUS, Web of Science, and Embase. From this common methodology, discoveries and findings were identified and summarized in this final review.

\section{Detailed classification}

The probability of relative impact between the adverse event and the drug is usually estimated by the physicians or clinical pharmacologists/pharmacists in a hospital setting, as they are responsible for recognizing or suspecting most of the ADRs during treatment [12]. The causality assessment is the responsibility of either a single expert or an established committee. As it is a common phenomenon of variable perception of knowledge and experience by each expert, there is a high possibility of disagreement and interindividual variability on assessment. Various methods of causality assessment are described here.

\section{Swedish method by Wilholm et al. 1984}

Swedish method was confined to the regulatory agency of Sweden. Seven factors are considered by the clinician to assess the causal relationship: (i) the time sequence, (ii) previous medication history, (iii) dose correlation, (iv) The pattern of response to drugs, (v) drug rechallenge, (vi) alternative causes for etiology and (vii) concurrent medications received. Events due to drugs are allocated as probable or possible and non-assessable or unlikely.

Limitation: Huge chance of overlap and evaluation errors of ADRs can be occurred due to low categorical choices of assessment of causality is the considerable limitation for this method [25]

\section{Dangaumou's french method}

French regulatory agency used this method since 1977 . The method uses two different tables containing three chronological and four semiological criteria's. The chronological/sequential principles are (i) challenge of drugs, (ii) dechallenge and (iii) rechallenge, with a comprehensive score of four possible categories. The semiological principles include (i) Clinical signs present (ii) favoring elements, (iii) Alternative explanation not related to drugs (none or possible) and (iv) Three possible results (positive, negative or no test for the event-drug pair) to specific lab test. Assessment is classified into likely, possible and dubious.

The stated method has the benefit of separate analysis of each drug and it grants certain drugs at a time excluding the suspected drug.

Limitation: The only limitation of the stated method is it consumes more time and analysis compared to alternative algorithms [26].

\section{Kramer et al. 1974 method}

This method is applicable for the assessment of single suspect drugs and in the case of multiple drug administration, each one is separately assessed. Kramer's method is known for its transparency.

Limitation: Effective utilization of this method requires certain levels of experience, time and expertise [27].

\section{Balanced assessment method (Lagier et al. 1983)}

Evaluation can be done for case reports considering the likelihood of each criterion. Consideration of the possibility for alternative causes of each factor is the main advantage of this scale. Two independent assessors need to evaluate this scale; however, the evaluation still varies based on their level of expertise and knowledge.

Limitation: Assessors should be expertise in the relevant field to make efficient and determined evaluation [28].

\section{Summary time plot (Castle et al. 1984)}

To identify patterns and incidence of ADRs, this method was recommended for the industrial context. It summarizes the plausible relationship between event and drug based on time factor. After analyzing enough information, a plot is made with the onset lag time of ADR and drug on X-axis and ADR severity on Y-axis. This method saves time and understandable with legible terminology and provides reliable results with minimal information.

Limitation: This method majorly considers and summarizes the time factor and cannot provide conclusions based on other factors [29]

\section{Cibageigy method (Venulet et al.1980)}

After several consensus meetings, this method was proposed. Initially, this method was based on an analogous visual scale, later a checklist of 23 questions with three parts has been updated to this technique. (i) Current ADR History (ii) Previous ADR experience of a patient (iii) Consulting clinician experience. Compared to the initial Visual Analogue (VA) Scale (based on evaluator's assessments), the updated method has a high acceptance rate.

Limitation: Though the results are reliable, to assure validity of the knowledge and expertise of the evaluator reflect in the assessment [30].

\section{Loupi et al. 1986 method}

This method is applicable in the assessment of the teratogenic potential of the drug. The Algorithm has been categorized into two sections on an axial basis. The first section allows excluding the drug if not plausible for the abnormality whereas the second section relies on the bibliographical data.

Limitation: To conclude the results, alternative etiological reasons are being considered by three questions from the two sections irrespective of suspected drug and bibliographic data [31].

\section{Maria and victorino ( $M$ and $V$ ) scale}

To diagnose drug-induced liver injury (DILI), Maria and Victorino developed this scale. Causal relationship was categorized as a score between 6 and 20 (score of $>17$, definite; 14-17, probable; 10-13, possible; 6-9, unlikely; $<6$, excluding hepatotoxic drugs). To acquire accurate results, it requires clinical expertise as the diagnosis of DILI is complex. Evaluation should be done separately for each drug in case of multiple suspected drugs.

Limitation: It is difficult to generate scores for all types of DILI, as some questions are applicable only for immune allergic hepatitis in this scale [32].

\section{Australian method}

Australian method concludes results from internal data available in the case reports on timing and laboratory findings. Deliberate exclusion of previous knowledge on the suspected drugs was done in the assessment.

Limitation: Limitation of this method is the likelihood of causal relationships [33].

\section{World Health Organization (WHO)-Uppsala Monitoring Centre (UMC) causality assessment criteria}

WHO causality assessment scale is majorly used scale for the assessment of the causal relationship of case reports and has been developed during the International Drug Monitoring Programme in discussion with national centers.

This scale has been categorized into 6 groups considering the basic criteria of 4 requirements in each category. These 4 criteria include a) temporal relationship b) plausibility and absence of other factors c) laboratory findings and d) de-challenge and re-challenge. Unclassified is applicable when additional information is necessary to evaluate the relationship [11].

\section{Naranjo scale}

Naranjo scale assesses the causality using the traditional categories of definite, probable, possible and doubtful. A ten elemental questionnaire with yes, no and unknown replies are developed. Based on the replies, the score has been determined into categories.

Limitation: The Naranjo Scale does not address the points needed in the assessment of the causality of possible drug interactions [34]. 


\section{Drug interaction probability scale (DIPS)}

Horn et al. proposed a DIPS scale to evaluate ADRs caused due to drug interactions. To estimate the likelihood of drug interactions, DIPS adopted ten questions with 'yes' or 'no' answers yielding a score. DIPS covers the information on pharmacological properties of the drug, patient profile and impact of other drugs through the questions.

Limitation: Assessment of this scale can be done only by experts who have sound knowledge in pharmacological and pharmacokinetic profiles of involved drugs [35].

\section{Bayesian adverse reactions diagnostic instrument (BARDI)}

To overpower the limitations of variability with skilled judgments and algorithms, the BARDI method was developed. This method evaluates posterior odds in favor of suspected drugs over other alternate factors. Posterior odd factor considers six subsets of assessment; the first subset (prior odds) deals with epidemiological data, clinical trial information and population pharmacokinetics, whereas the rest of the five subsets (likelihood ratios) deal with specific case report information.

Either on paper or computer, this method can be demonstrated as a spreadsheet Programme. Instant feedbacks in numerical and graphical values are being calculated as soon as new evidence of suspected ADR is assessed. Case parameters of case findings and scoring are calculated in the spreadsheet by using the software. During the process, the spreadsheet allows rapid calculations.

Limitation: Sometimes the results provide multiple causal associations with drug and event, even though operated by experts [36].

\section{MacBARDI spreadsheet}

The computerized MacBARDI spreadsheet includes or needs five kinds of data: (i) Pure lines of data describing the required input; (ii) Input lines that are used as calculation criteria for each of the six variables; (iii) Assumption lines that are integrated inputs used in calculations; (iv) Lines of calculation for calculating and showing the significance of each term in the evaluation; (v) Output lines showing the value of each aspect needed to measure the posterior odds and the posterior evens itself. MacBARDI enables case analyses to be updated as and when fresh data is available. This method has all the necessary criteria for assessing causality (e. g. explicitness, flexibility), Promotes learning and modeling and significantly reduces the time needed to estimate cases. Other-Bayesian Adverse Reactions Diagnostic Instrument (BARDI) prototypes created for the diagnosis of ADRs comprise a risk forecast exemplary for pseudoallergic response and release of Histamine in patients experiencing surgery [31] as well as diagnostic assistance for pseudomembranous colitis [30]. The merits of BARDI are reliability (the same input information brings out the same output) explicitness and transparency (final results show undoubtedly what information is considered and its contribution in the evaluation) and an etiological balancing (all drug and non-drug possible causes are considered in the evaluation).

Limitation: This approach's apparent limitations are the significant amount of time, resources and complex calculations involved [37].

\section{Causality assessment of vaccine-related adverse events}

Vaccines require a high degree of safety and efficacy strategies as these are administered in healthy individuals, especially in neonates and infants for beneficiary outcomes. Though most of the adverse events due to vaccines are unavoidable, causality assessment has to be given utmost priority. Advisory Committee on Causality Assessment (ACCA) in Canada developed a method for vaccine-related events. ACCA receives the most unusual and serious adverse events due to vaccines. ACCA reviews every individual case in a methodological and systematic way to evaluate causal association on a form using specialists from various clinical and medical departments. This form consists of seven sections that focus on different parameters related to the importance of adverse reactions due to vaccine and its impact for further evaluation [38].

\section{Karch and lasagna scale}

Karch and lasagna scale have been made known in the early 1970s and have a correlation to that of the WHO causality scale. Causality has been classified as definitive, probable, possible, conditional or unlikely. It has not been featured as there are no distinct advantages compared to other scales. Some of the studies had been conducted and the results of the studies found attributed to karch and lasagna scale over the WHO-UMC scale.

Limitation: Duplicability and validity of results are not well established which will influence the quality of reports for further validation [39].

\section{Begaud algorithm}

Begaud algorithm has been executed in France during the 1970s and evaluation depends on the three-stage process. It collaborates chronological and symptomatological evaluations to obtain a 3degree global score with principles of doubtful, possible and probable.

Limitation: Begaud method deals with each drug as an individual case. Combinations of drugs cannot be taken into consideration. Reintroduction of the same drug or drug of the same class under various circumstances/conditions is not taken into as real rechallenge [40].

\section{Hallas scale}

Although evaluating and identifying the ADRs is complicated, estimating the avoidability of ADRs in the course of therapy is further challenging. Hallas scale is a sequence of four statements concerning the estimation of avoidability of associated ADRs with drugs. This scale can be advantageous not only to determine how many degrees an ADR can be avoidable but also to evaluate treatment adherence towards standard guidelines.

Limitation: For inpatients and therapy procedures where conditions are highly variable and chronic, the Hallas scale cannot be applicable. Thorough knowledge of treatment procedures with uninterrupted updating is needed to meet the criteria standards where it is dynamically changing. This makes the Hallas scale irreproducible and less reliable [41].

\section{PRISCUS list}

PRISCUS List includes the list of potentially inappropriate medications (PIM) for geriatric patients. It consists of instructions for clinical practice and therapeutic alternatives. PRISCUS list was generated step by step of quality analysis, literature search, and development of preliminary PIM list. This preliminary list finally altered utilized Delphi method for validation.

Limitation: Little scientific data is available for assessment of recommendations and alternate approaches. Validity and practicability are still to be developed of PRISCUS List as it is restricted to the German epidemiological scenario [42].

\section{RUCAM scale}

The Roussel Uclaf Causality Assessment Method (RUCAM) is extensively used to evaluate the causal relationship between drugs and hepatic injury. It can be utilized for objective assessment of hepatotoxic drugs under development in the clinical trial settings.

Limitation: Scoring system does not incorporate factors of age, alcohol dependence and other factors that diverge the results of the causal relationship of drugs with hepatic injury. It should be contrived in addressing the uncertainty of results eliminating other factors that cause liver injury [43].

\section{CIOMS scale}

Council for International Organizations of Medical Sciences (CIOMS) scale has been contemplated as a standard causality assessment tool for Drug and herb induced liver injuries. The CIOMS scale has been recommended by eight scientists from 6 countries of hepatology specialization during a consensus meeting. Numerous advantages in using the CIOMS scale with its high specificity and validation regarding hepatotoxicity and its causative source with positive re- 
exposure test. This scale meets all the criteria for a valid causality assessment procedure for prospectively collected cases.

Limitation: Risk factors for liver injury have been restricted to only alcoholism and age in CIOMS scale. Additional assistance to provide quality data in case of retrospective case collection. It has a very limited exclusion of other causes of hepatotoxicity [44].

\section{Liverpool ADR scale}

Gallagher et al. have developed the Liverpool ADR causality assessment tool depending on the criteria given by Sir Branford Hill. They demonstrated compatibility results with a few variable controversies with the Naranjo scale and high inter-rater reliability (IRR). This scale has a flow diagram arrangement rather than a scoring system, which makes to evaluate easier and quicker.

Limitation: Validation of the tool has been done internally and not independently. This scale needs further validation and certainty of results by experts [45].

\section{Visual Analogue Scale (Miremont et al. 1994)}

Visual analogue scale has been proposed by Miremont et al. and compared the thoughts of physicians with a standardized French causality assessment scale. A study has been conducted on 75 ADR cases involving 120 drugs. In the course of study, physicians presented results of 'likely' and 'very likely' for $60 \%$ of cases and 'unlikely' or 'possible' for $32 \%$ of cases, whereas results of standard French causality model provided only $11 \%$ for 'likely' and $89 \%$ for 'possible' or 'unlikely'. Only $6 \%$ of cases are in complete accordance with both physicians and the standard methods.

Limitation: Rate of agreement and lack of precision in decision making and was lower for intermediate causality levels [46].

\section{Blanc et al.}

Blanc et al. developed a decision table in 1979 to evaluate the nature of relationships amidst drugs and events. Time sequence, response pattern and role of the underlying disease(s) are three considerable factors for this table. By incorporating these three factors, it has been created with a five option causality scale ranging from doubtful to certain. The results presented by this scale have given very low concordance and are not outstanding [17].

\section{Emanueli and sacchetti}

Emanueli and Sacheti have evolved an algorithm for the categorization of adverse events in clinical trials. This algorithm has been developed on the basic principle of the Karch and Lasagna scale. A 5 point scale decision table regulates the probability of the relationship between drug and event. Precision and reliability of results have confirmed to be higher and utilized in many large scale clinical trials.

Limitations: It cannot rule out other causes like clinical state and other therapies, which makes it difficult to evaluate events that fall under the class of higher than possible [47].

\section{Stephens algorithm}

In 1984, Myles Stephens proposed the indicated algorithm to evaluate drugs that are under both pre-marketing and postmarketing surveillance. It can also differentiate the suspected drugs from various concurrent drugs in assessing causality. It has been developed based on the criteria of kramer's and Naranjo Scale [48].

\section{CONCLUSION}

Many of the causality assessment methods have their own merits and demerits. The purpose of the development of standard ADR assessment methods is to implement reliable, reproducible and validated information regarding the association of adverse reactions and suspected drugs. Some of the scales may serve their purpose to a major extent; however, no single scale has been adopted as standardized and considered for uniform acceptance. However, WHO causality assessment scale and Naranjo probability scale are the widely used and commonly preferred in most of the practices.

\section{ABBREVIATIONS}

ADR-Adverse Drug Reaction, VA-Visual Analogue, DILI-Drug Induced Liver Injury, WHO-World Health Organization, UMCUppsala Monitoring Center, DIPS-Drug Interaction Probability Scale, BARDI-Bayesian Adverse Reactions Diagnostic Instrument, ACCAAdvisory Committee on Causality Assessment in Canada, PIMPotentially Inappropriate Medications, RUCAM-Roussel Uclaf Causality Assessment Method, CIOMS-Council for International Organizations of Medical Sciences, IRR-High Inter-Rater Reliability

\section{ACKNOWLEDGMENT}

Authors want to acknowledge the immense help obtained from the scholars whose articles are cited for this reference article.

\section{FUNDING}

\section{Nil}

\section{AUTHORS CONTRIBUTIONS}

Dr. A. Pramod Kumar conducted the search and collected the reference articles; Dr. Dharini and Haripriya wrote the manuscript; Dr. Ch. Sri Harsha reviewed the manuscript.

\section{CONFLICTS OF INTERESTS}

The authors declare no conflicts of Interest

\section{REFERENCES}

1. Rehan HS, Chopra D, Kakkar A. Physician's guide to pharmacovigilance: terminology and causality assessment. Eur J Intern Med 2009;20:3-8.

2. Sangeetha RA, Jamuna RR, Kala P. Pattern of adverse drug reactions in a tertiary care teaching hospital: a cross-sectional study. Asian J Pharm Clin Res 2017;10:170-3.

3. Shareef JA, Fernandes JE, Samaga LA, Khader SA. A study on adverse drug reactions in hospitalized patients with diabetes mellitus in a multi-specialty teaching hospital. Asian J Pharm Clin Res 2016;9:114-7.

4. Lazarou J, Pomeranz BH, Corey PN. Incidence of adverse drug reactions in hospitalized patients: a meta-analysis of prospective studies. JAMA 1998;279:1200-5.

5. Ramesh M, Pandit J, Parthasarathi G. Adverse drug reactions in a south Indian hospital-their severity and cost involved. Pharmacoepidemiol Drug Saf 2003;12:687-92.

6. Arulmani R, Rajendran SD, Suresh B. Adverse drug reaction monitoring in a secondary care hospital in South India. Br J Clin Pharmacol 2008;65:210-6.

7. Alhawassi TM, Krass I, Bajorek BV, Pont LG. A systematic review of the prevalence and risk factors for adverse drug reactions in the elderly in the acute care setting. Clin Interv Aging 2014;9:2079-86.

8. Bord CA, Rachl CL. Adverse drug reactions in United States hospitals. Pharmacother: J Human Pharmacol Drug Ther 2006;26:601-8.

9. Kongkaew C, Noyce PR, Ashcroft DM. Hospital admissions associated with adverse drug reactions: a systematic review of prospective observational studies. Ann Pharmacother 2008;42:1017-25.

10. Smyth RL, Peak M, Turner MA, Nunn AJ, Williamson PR, Young $\mathrm{B}$, et al. Systematic review of paediatric adverse drug reactions. InADRIC: Adverse Drug Reactions In Children-a programme of research using mixed methods. NIHR Journals Library; 2014.

11. World Health Organization (WHO), Uppsala Monitoring Centre. The use of the WHO-UMC system for standardized case causality assessment. WHO [Internet]. Available from: http://www.who-umc.org/graphics/4409.pdf. [Last accessed on 10 Jan 2020].

12. Macedo AF, Marques FB, Ribeiro CF, Texeira F. Causality assessment of adverse drug reactions: comparison of the results obtained from published decisional algorithms and from the evaluations of an expert panel. Pharmacoepidemiol Drug Saf 2005;14:885-90. 
13. Arimone Y, Begaud B, Miremont Salame G, Fourrier Reglat A. Agreement of expert judgment in causality assessment of adverse drug reactions. Eur J Clin Pharmacol 2005;61:169-73.

14. Turner WM. The food and drug administration algorithm. Special workshop-regulatory. Drug Inf J 1984;18:259-66.

15. Danan G, Benichou C. Causality assessment of adverse reactions to drugs-I. A novel method based on the conclusions of international consensus meetings: application to druginduced liver injuries. J Clin Epidemiol 1993;46:1323-30.

16. Blanc $S$, Leuenberger $P$, Berger JP, Brooke EM, Schelling JL. Judgments of trained observers on adverse drug reactions. Clin Pharmacol Ther 1979;25:493-8.

17. Taofikat BA, Savovi J, Ernst E. Methods for causality assessment of adverse drug reactions a systematic review. Drug Safety 2008;31:21-37.

18. Meyboom RH, Egbertes ACG, Gribnau FW, Hekster YA. Pharmacovigilance in perspective. Drug Safety 1999;21:42947.

19. Macedo AF, Marques FB, Ribeiro CF, Teixeira F. Causality assessment of adverse drug reactions: comparison of the results obtained from published decisional algorithms and from the evaluations of an expert panel, according to different levels of imputability. J Clin Pharm Ther 2003;28:137-43.

20. Rodriguez MC, Vacca CP, Perez J, Bignone I, Bergman M, Valsecia M. Buenas practicas de Farmacovigilancia para las Americas. Washington DC. Organización Panamericana de la Salud; 2011. p. 73

21. Shakir SAW, Layton D. Causal association in pharmacovigilance and pharmacoepidemiology thoughts on the application of the Austin Bradford-Hill criteria. Drug Safety 2002;25:467-71.

22. Mugosa S, Bukumiric Z, Kovacevic A, Boskovic A, Protic D Todorovic Z. Adverse drug reactions in hospitalized cardiac patients: characteristics and risk factors. Vojnosanitetski Pregled 2015;72:975-81

23. Meyboom RH, Hekster YA. Causal or casual? The role of causality assessment in pharmacovigilance. Drug Safety 1997;17:374-89.

24. Angamo MT, Chalmers L, Curtain CM, Bereznicki LR. Adversedrug-reaction-related hospitalisations in developed and developing countries: a review of prevalence and contributing factors. Drug Safety 2016;39:847-57.

25. Wiholm BE. The swedish drug-event assessment methods. Special workshop-regulatory. Drug Inf J 1984;18:267-9.

26. Dangoumau J, Evreux JC, Jouglard J. Method for determination of undesirable effects of drugs. Therapie 1978;333:73-81.

27. Kramer MS, Leventhal JM, Hutchinson TA, Feinstein AR. An algorithm for the operational assessment of adverse drug reactions: Background, description, and instructions for use. JAMA 1979;242:623-32.

28. Lagier G, Vincens M, Castot A. Imputability in drug monitoring: principles of the balanced drug reaction assessment method and principal errors to avoid. Therapie 1983;38:303-18.

29. Castle WM. Assessment of causality in industrial settings. Drug Inf J 1984:18:297-302.

30. Venulet J, Ciucci A, Berneker GC. Standardised assessment of drug-adverse reaction associations: rationale and experience. Int J Clin Pharmacol Ther Toxicol 1980;18:381-8.
31. Loupi E, Ponchon AC, Ventre JJ, Evreux JC. Imputability of a teratogenic effect. Therapie 1986;41:207-10.

32. Maria VA, Victorino RM. Development and validation of a clinical scale for the diagnosis of drug-induced hepatitis. Hepatol 1997;26:664-9.

33. Mashford ML. The Australian method of drug-event assessment. Special Workshop-regulatory. Drug Inf J 1984;18:271-3.

34. Naranjo CA, Busto U, Sellers EM, Sandor P, Ruiz I, Roberts EA, et al. A method for estimating the probability of adverse drug reactions. Clin Pharmacol Ther 1981;30:239-45.

35. Horn JR, Hansten PD, Chan LN. Proposal for a new tool to evaluate drug interaction cases. Ann Pharmacother 2007;41:674-80.

36. Naranjo CA, Lanctot KL. A consultant"s view on the role of Bayesian differential diagnosis in the safety assessment of pharmaceuticals. Drug Inf J 1992;26:593-601.

37. Lanctot KL, Naranjo CA. Computer-assisted evaluation of adverse events using a Bayesian approach. J Clin Pharmacol 1994;34:142-7.

38. Collet JP, Macdonald N, Cashman N, Pless R. Monitoring signals for vaccine safety: the assessment of individual adverse event reports by an expert advisory committee. Bull World Health Organisation 2000;78:178-85.

39. Kyonen M, Folatre I, Lagos X, Vargas S. Comparison of two methods to assess causality of adverse drug reactions. Rev Med Chile 2015;143:880-6.

40. Benahmed S, Picot MC, Dumas F, Demoly P. Accuracy of a pharmacovigilance algorithm in diagnosing drug hypersensitivity reactions. Arch Intern Med 2005;165:1500-5.

41. Smyth RL, Peak M, Turner MA, Nunn AJ, Williamson PR, Young $\mathrm{B}$, et al. Development of the liverpool adverse drug reaction avoidability tool. In ADRIC: Adverse drug reactions in childrena programme of research using mixed methods. NIHR Journals Library; 2014.

42. Holt S, Schmiedl S, Thürmann PA. Potentially inappropriate medications in the elderly: the PRISCUS list. Dtsch Arztebl Int 2010;107:543.

43. Lewis JH, Larrey D, Olsson R, Lee WM. Utility of the roussel uclaf causality assessment method (RUCAM) to analyze the hepatic findings in a clinical trial program: evaluation of the direct thrombin inhibitor ximelagatran. Int J Clin Pharmacol Ther 2008;46:327-39.

44. Teschke R, Wolff A, Frenzel C, Schwarzenboeck A. Drug and herb induced liver injury: council for international organizations of medical sciences scale for causality assessment. World J Hepatol 2014;6:17.

45. Gallagher RM, Kirkham JJ, Mason JR, Bird KA. Development and inter-rater reliability of the Liverpool adverse drug reaction causality assessment tool. S PLos One 2011;6. Doi:10.1371/journal.pone.0028096

46. Miremont G, Haramburu F, Begaud B, Pere JC. Adverse drug reactions: physicians' opinions versus a causality assessment method. Eur J Pharmacol 1994;46:285-9.

47. Emanueli A, Sacchetti G. An algorithm for the classification of untoward events in large scale clinical trials. Agents and actions. Supplements 1980;7:318-22.

48. Stephens M. Assessment of causality in industrial setting special workshop industrial. Drug Inf J 1984;18:307-13. 